\title{
Three-dimensional Flow Measurements around Micro-pillars Made by UV-NIL in Water via Micro-digital Holographic Particle Tracking Velocimetry (Micro-DHPTV)
}

\author{
Yasuhiro Matsuda ${ }^{1}$, Hiroshi Kigami ${ }^{1}$, Noriyuki Unno ${ }^{2}$, \\ Shin-ichi Satake ${ }^{1}$, and Jun Taniguchi ${ }^{*}$ \\ ${ }^{1}$ Department of Applied Electronics, Tokyo University of Science, \\ 6-3-1 Niijuku, Katsushika-ku, Tokyo 125-8585, Japan \\ ${ }^{2}$ Department of Mechanical Engineering, Sanyo-Onoda City University, \\ Daigakudo-ri 1-1-1, Sanyo-Onoda, Yamaguchi 756-0884, Japan \\ *junt@te.noda.tus.ac.jp
}

\begin{abstract}
A refractive index matching method in water to measure the velocity field around micropillars was investigated. The micro-pillars were fabricated from an optical material whose refractive index was 1.333, which is the same as that of water. The fabrication method used was UV imprint lithography (UV-NIL) with photolithography with a replica mold. The moving tracer particles on the pillars were measured by micro-digital holographic particle tracking velocimetry (micro-DHPTV). Because the refractive indices of the pillars and water were the same, the hologram images could be obtained clearly without diffraction around the micro-pillars. As a result, tracking of particles and measurement of velocity fields using reconstructed particles could be achieved without optical distortion. The moving particles could be captured around the micro-pillars.
\end{abstract}

Keywords: Holography, Index matching method, Particle tracking velocimetry, Micropillar

\section{Introduction}

Microfluidic devices [1-4] are expected to be a promising technology for next generation biotechnology, such as micro-total analysis systems ( $\mu$-TAS) [5]. In micro-channels, however, it is difficult to utilize turbulence and inertial effects because the Reynolds numbers of micro-channels tend to be low [6]. To clarify and design the fluid flow, therefore, techniques for three-dimensional velocity measurements are required to detect flow fields in the microfluidic devices used in bioresearch. In a previous study, an investigation into the mechanism of actin network accumulation was carried out by measuring flow fields in a microdevice with a pattern of polydimethylsiloxane (PDMS) micro-pillars [7]. The flow field was obtained by digital inline holographic microscopy (DIHM) [8]. However, particle tracking was difficult because of the optical distortion resulting from the difference between the refractive indices of water and PDMS. Given that water is generally used as the working fluid in biofluidics, the refractive index of MY-133-V2000 (MY Polymers Ltd., NesZiona, Israel) can match that of water, so that obstacles caused by optical distortion, such as those observed with PDMS, can be avoided. In past researches, fluid measurements for microflow [9] and nanoflow [10] were successfully achieved via a refractive index matching method [11] using MEXFLON, an optical material whose refractive index matches that of water. In addition, MY-134 (MY Polymers Ltd., Nes-Ziona, Israel, $n=1.34$ ) an ultraviolet (UV)-curable resin [12], is a potential refractive index matching material. In a previous study, a cell on the micro-pillar fabricated by the material in the cell culture liquid $(n=1.338)$ was observed. Additionally, the calibration plate was fabricated for multilayer nanoparticle image velocimetry using UV nanoimprint lithography (UV-NIL) $[13,14]$ with the low optical index UV 
resin (MY-133-V2000) [15]. The fabrication process involved the use of photolithography and UV-NIL with a replica mold. In the present study, we measured three-dimensional flow fields around a micro-pillar prepared by the abovementioned technique.

\section{Experimental}

2.1. Fabrication of a micro-pillar using low optical index UV resin (MY-133-V2000)

The fabrication of the replica mold is illustrated in Fig. 1. First, a master mold with pillar patterns was fabricated by photolithography. Then, the UVcurable resin (PARQUIT OEX-028-X433-3, hereafter X433-3; AUTEX Co., Ltd., Tokyo) was positioned on the master mold. Afterward, the resin layer was covered with a polyester film (Cosmoshine A4300; Toyobo Co. Ltd., Osaka) (Fig. 1(a)), pressed at $0.3 \mathrm{MPa}$ (Fig. 1(b)), and then cured using UV radiation (Fig. 1(c)). After curing, the polyester film was released from the master mold (Fig. 1(d)) and baked at $85{ }^{\circ} \mathrm{C}$ for $30 \mathrm{~min}$ to induce the required antifouling effect (Fig. 1(e)) [16]. Using the replica mold, pillar patterns of the low optical index UV resin were fabricated on a cleaned glass slide (Micro cover glass No.1, Matsunami Glass Inc., Ltd.) by UV-NIL in atmospheric or vacuum environment.

The UV-NIL process was similar to that illustrated in Fig. 1, that is, the transfer resin was the low optical index UV resin, the patterned mold was the replica mold, and the support substrate was a glass slide (Fig. 1(a)). Other UV-NIL conditions were as follows: UV dose of the low optical index $\mathrm{UV}$ resin, $10 \mathrm{~J} / \mathrm{cm}^{2}$; imprint pressure, $8 \mathrm{MPa}$; and pressure of the vacuum environment, $<1 \mathrm{~Pa}$. However, the adhesive force between the low optical index UV resin and the cleaned glass slide was very weak; therefore, an adhesive layer was required.

(a) Dropping $X 433-3$

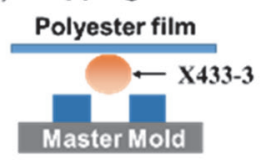

(d) Release

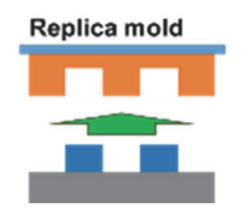

(b) Pressed

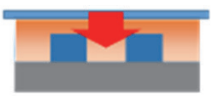

(e) Baking

$85^{\circ} \mathrm{C}$ $30 \mathrm{~min}$

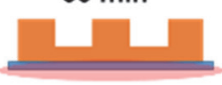

(c) UV curing

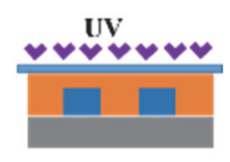

(d) Separating (LTIL)

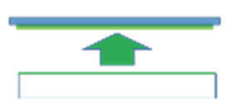

Fig. 2. Fabrication process of the thin adhesive layer on the glass slide.

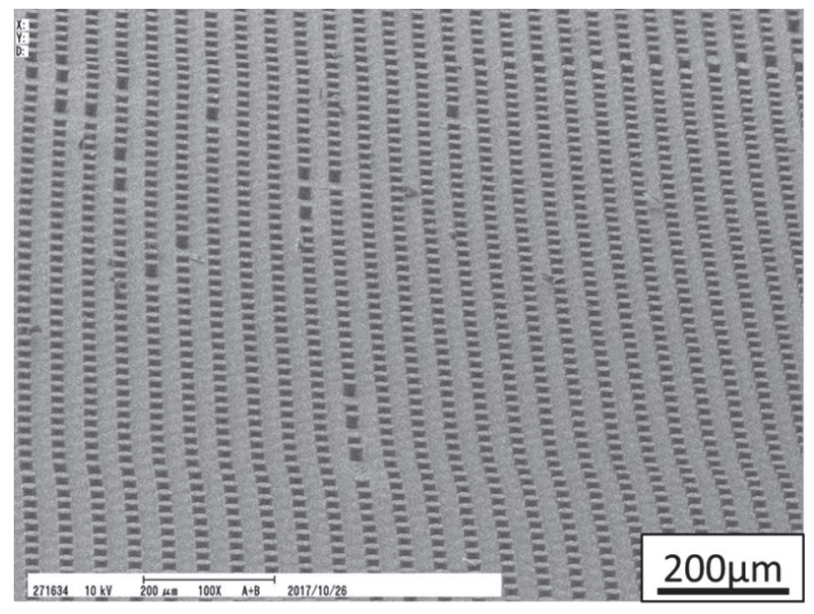

Fig. 3. SEM image of the microstructures fabricated from the low optical index UV resin by the vacuum UV imprinting technique.

\section{(e) UV curing}

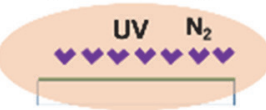

Fig. 1. Fabrication of the replica mold.

Figure 2 illustrates the method for coating the adhesive layer on the glass slide. First, photo-curing resin PAK-02 (Toyo Gosei Co. Ltd., Tokyo) was dropped on the glass slide (Fig. 2(a)) and spread using a doctor blade with a blade speed of 25 $\mathrm{mm} / \mathrm{min}$ (Fig. 2(b)). To reduce the adhesive layer thickness, a liquid transfer method [17] was used. A polyester film was used to cover the thin PAK-02 layer on the glass slide prepared by the doctor blade method, and then, the film was roll-pressed under a force of $900 \mathrm{~N}$ and rotating speed of $1.6 \mathrm{~mm} / \mathrm{s}$ (Fig. 2(c)). Subsequently, the polyester film was separated from PAK-02 on the glass slide surface. Excess PAK-02 resin was captured on the polyester film and thus removed from the glass slide (Fig. 2(d)) [18]. The thin PAK-02 film was cured under a UV dose of $10 \mathrm{~mJ} / \mathrm{cm}^{2}$, in an ambient nitrogen atmosphere (Fig. 2(e)). The thickness of the PAK02 adhesive layer was less than $30 \mathrm{~nm}$. To compare different refractive indices, micro-pillars using the same mold pattern were fabricated from PAK-01 (Toyo Gosei Co. Ltd., Tokyo). Figure 3 shows a micro-pillar pattern imaged by scanning electron microscopy (SEM).
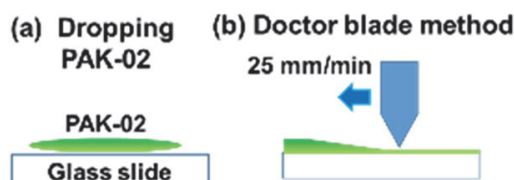

(c) Roll press

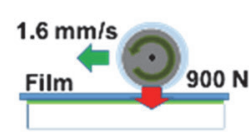


Figure 4 shows an expanded SEM image extracted from Fig. 3. The size of a pillar structure is also shown.

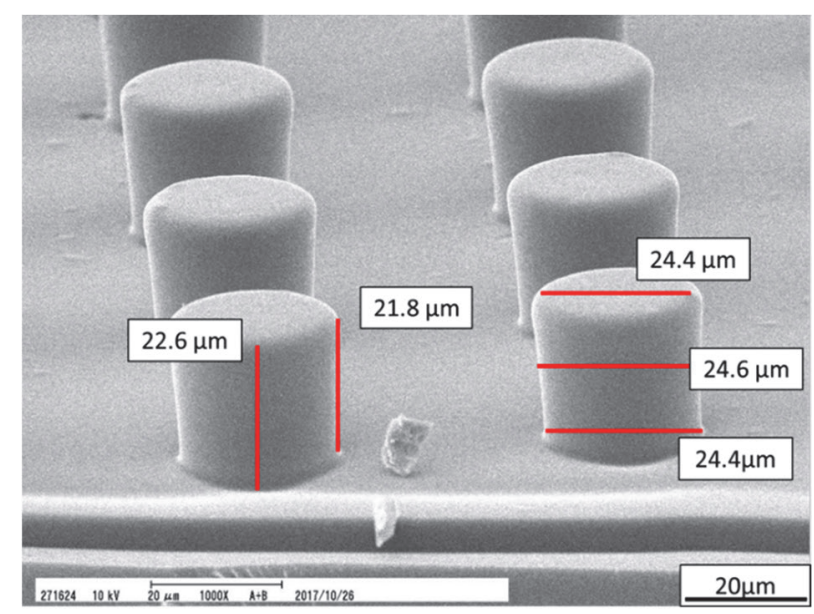

Fig. 4. SEM image of the microstructures fabricated from the low optical index UV resin by the vacuum UV imprinting technique (enlarged view).

2.2. Micro-DHPTV on the micro-pillar using the low optical index UV resin

Figure 5 shows the experimental setup. The equipment was the same as that used in our previous study [19].

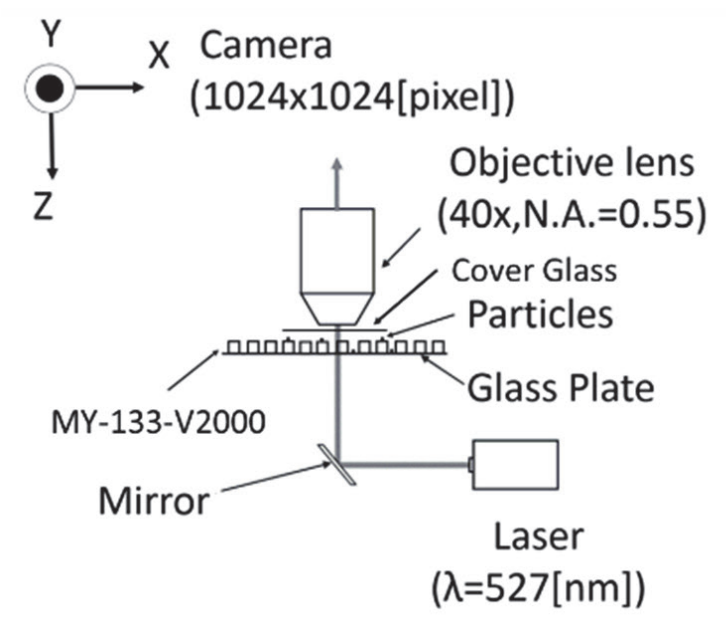

Fig. 5. Experimental setup for a micro-DHPTV system.

The plate was covered by glass and had micropillars made of the low optical index UV resin, whose refractive index is 1.333. A high-resolution digital camera (Redlake, MotionProX-3; resolution $1024 \times 1024$ pixel, image $12 \mu \mathrm{m} \times 12 \mu \mathrm{m}$ ) with an objective lens (Nikon, $40 \times, \mathrm{NA}=0.55$ ) was used to record fringe images of the particles. A neodymiumdoped yttrium lithium fluoride (Nd:YLF) laser (Photonics Industries DS20-527, $\lambda=527 \mathrm{~nm}$ ) was used as the light source, which produced a pair of laser pulses at a repetition rate of $1 \mathrm{kHz}$ with a pulse length of $58 \mathrm{~ns}$ and a pulse delay of $100 \mu \mathrm{s}$. The polystyrene spherical particles (Thermo Fisher Scientific 4010A Polystyrene) had a diameter of 1 $\mu \mathrm{m}$ and specific gravity of $1.05 . \mathrm{NaCl}(10 \mathrm{mM})$ was added to the particle suspensions to enable solid contact between the particles and the micro-pillar pattern [20]. The refractive index of the $10 \mathrm{mM}$ $\mathrm{NaCl}$ solution is the same as that of water. Particle suspensions or $10 \mathrm{mM} \mathrm{NaCl}$ particle suspensions were dropped on the micro-pillar pattern, and the plate was covered by a cover glass. Hologram images were recorded at a repetition rate of $1 \mathrm{kHz}$. The particles were tracked by DHPTV [19].

Figure 6 shows the schematic view of the DHPTV process. First, an in-line hologram image was captured at the best focus position. Then, the recorded hologram image was reconstructed using a computer hologram algorithm [19]. At this time, the reconstructed depth in the $\mathrm{Z}$ direction was varied from the best focus plane to the glass plate. Next, reconstructed brightness curves were obtained at each pixel. Finally, the z-positions of particles were determined by the peak of the brightness curves.

Three-dimensional flow measurements are performed using two captured images with the time difference of $1 \mathrm{~s}$.

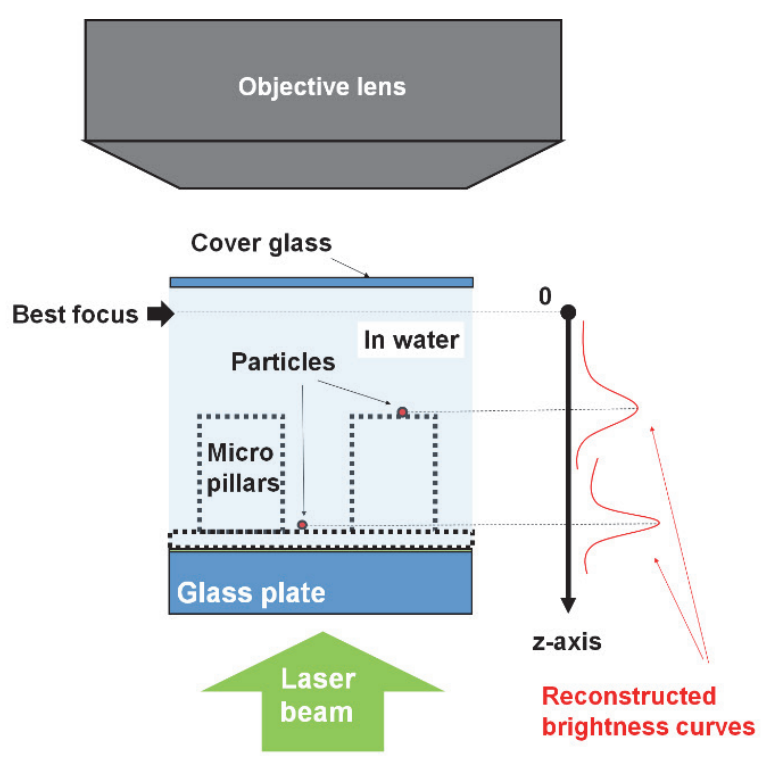

Fig. 6. Schematic view of the DHPTV process.

\section{Results and discussion}

Hologram images of the micro-pillar pattern are shown in Fig. 7. Figures 7(a) and 7(b) are obtained hologram images in air with the micro-pillar patterns of the low optical index UV resin and PAK- 
01, respectively. Figure 7(c) shows the micro-pillar pattern of the low optical index UV resin in the 10 $\mathrm{mM} \mathrm{NaCl}$ particle suspension, while Fig. 7(d) shows the micro-pillar pattern of PAK-01 in the particle suspension. The edge of the micro-pillar pattern of the low optical index UV resin became invisible as a result of refractive index matching when the pattern was immersed in the $10 \mathrm{mM} \mathrm{NaCl}$ particle suspension. On the other hand, the edge of the micro-pillar pattern of PAK-01 was visible even when the pattern was immersed in the particle suspension. It is clear, therefore, that the micropillar pattern of the low optical index UV resin can be used for refractive index matching with water. (a)

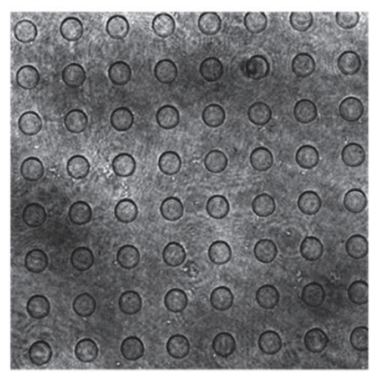

(c)

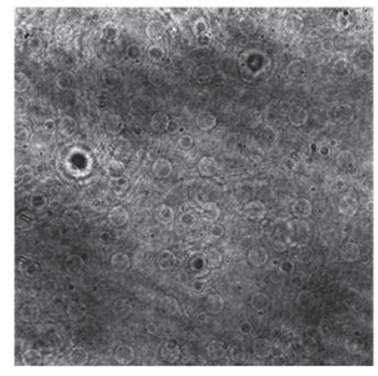

(b)

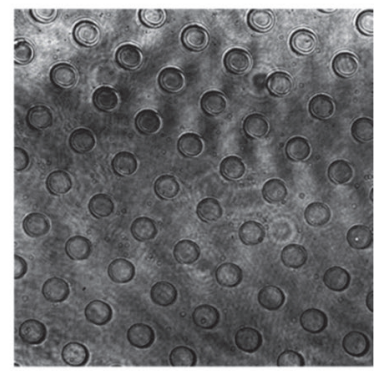

(d)

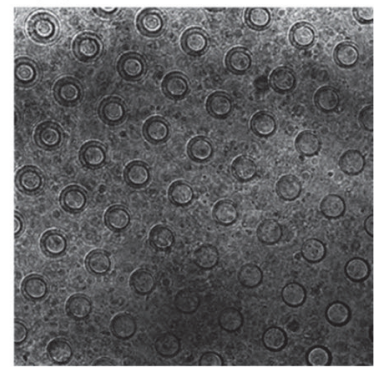

Fig. 7. Hologram images of microstructures (a) fabricated from the low optical index UV resin (dry), (b) fabricated from PAK-01 (dry), (c) fabricated from the low optical index UV resin (underwater), and (d) fabricated from PAK-01 (underwater).

Figure 8 shows a reconstructed image $60.1 \mu \mathrm{m}$ from the position of best focus, and particles $A$ and $\mathrm{C}$ placed on the ground of the micro-pillar pattern.

Figure 9 shows a reconstructed image $40.1 \mu \mathrm{m}$ from the position of best focus, and particles B and D placed on the top of micro-pillars.

Although the particles B and D were on micropillars and the particles $A$ and $C$ were close to micropillars, the reconstruction of the obtained hologram images was succeeded with the index matching method using the low optical index UV resin. Because the adhesive layer on the glass slide is planar and very thin of $30 \mathrm{~nm}$, in this study, the optical distortion was not observed.

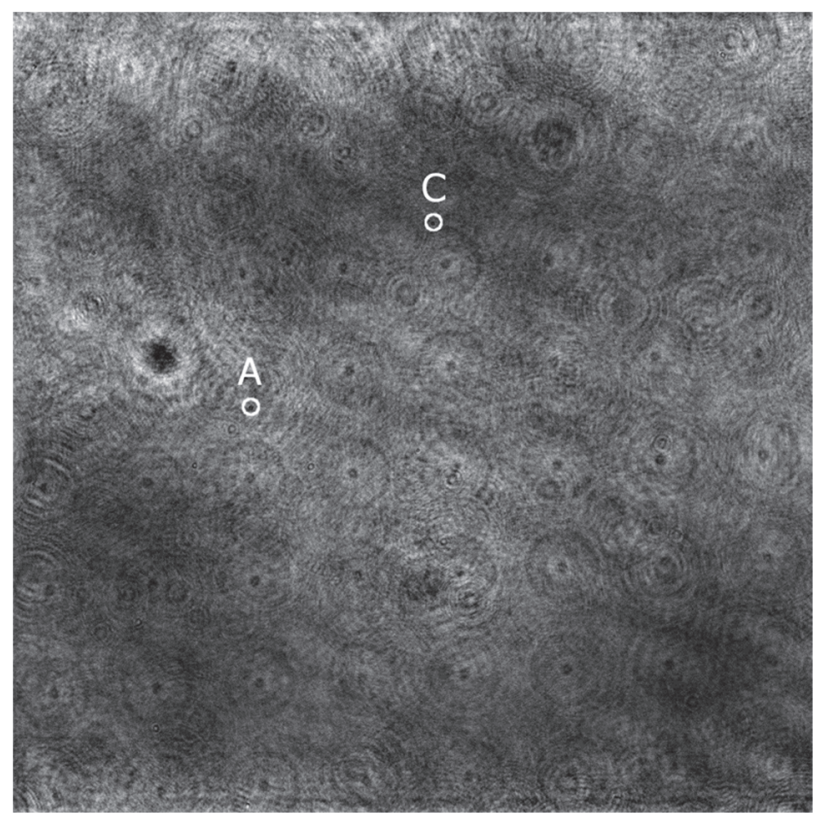

Fig. 8. Reconstructed image ( $60.1 \mu \mathrm{m}$ from best focus).

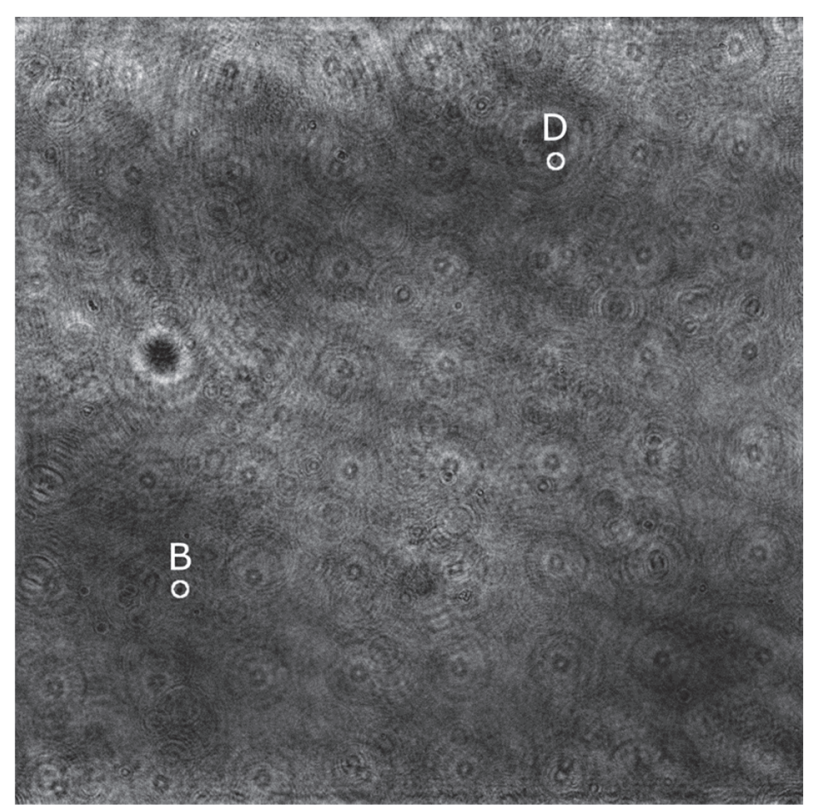

Fig. 9. Reconstructed image (40.1 $\mu \mathrm{m}$ from best focus).

Figure 10 shows the reconstructed longitudinal positions of particles A and B obtained from the depth with the highest brightness.

Figure 11 shows the reconstructed longitudinal position of particles $\mathrm{C}$ and $\mathrm{D}$ obtained from the depth with the highest brightness.

Table 1 shows the heights of the micro-pillars obtained from the difference in the depth of particles.

Figure 12 shows a three-view drawing of a reconstructed particle trajectory on the micro-pillar. The particle was reconstructed every $1.0 \mathrm{~s}$, and the 
micro-pillar was visualized only $5 \mu \mathrm{m}$ from the top. The motion of the particle on the micro-pillar was detected.

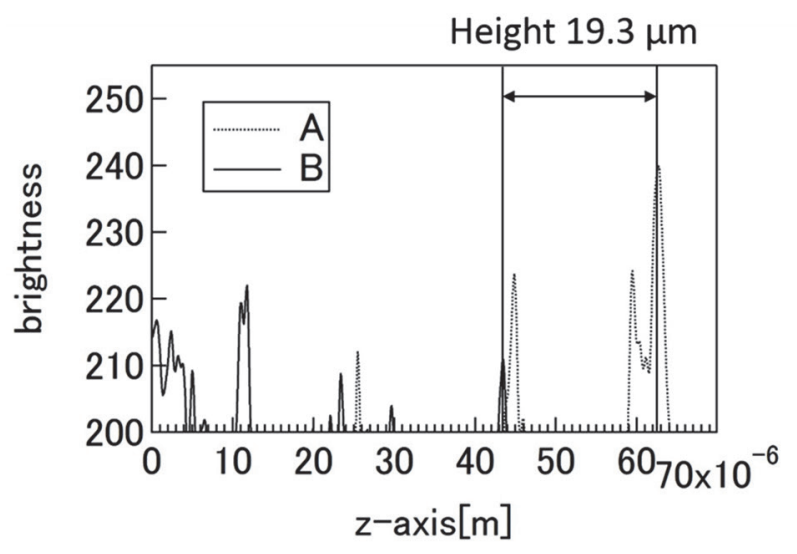

Fig. 10. Brightness of particles A and B.

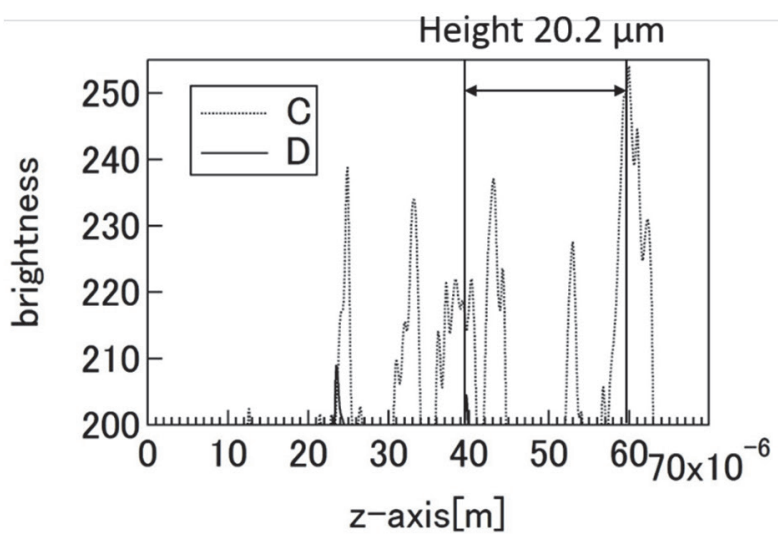

Fig. 11. Brightness of particles C and D.

Table 1. Heights of the micro-pillars.

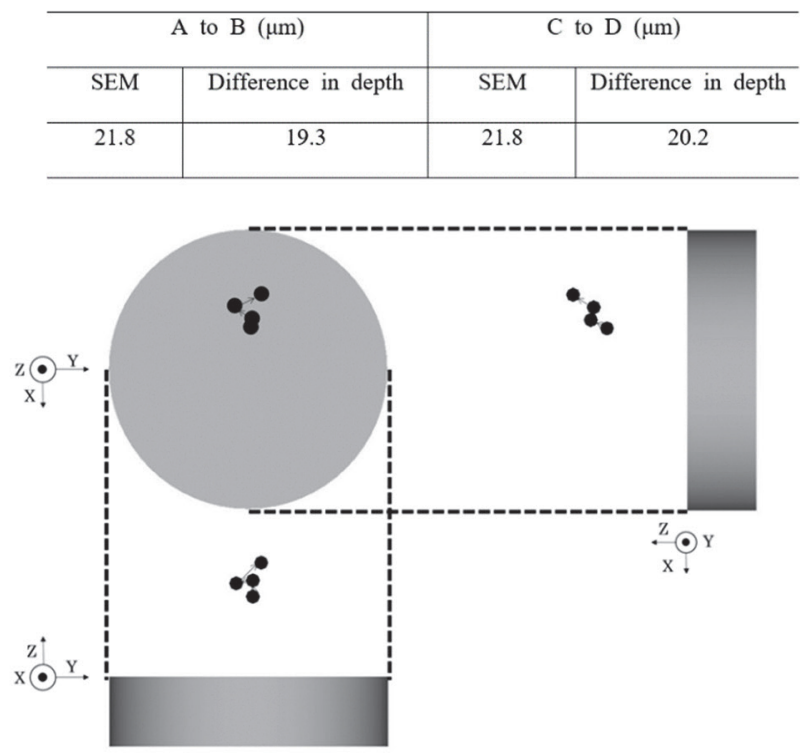

Fig. 12. Three-view drawing of reconstructed particle trajectory.

\section{Conclusion}

In this study, the micro-pillar pattern of the low optical index UV resin was fabricated, and refractive index matching was performed using the low optical index UV resin and water. The particle on the micro-pillar was reconstructed and tracked using micro-DHPTV. Refractive index matching with water will help in enhancing our understanding of the 3D flow around microstructures in water. We believe that our method will provide a deeper insight into the 3D flow around complex microstructures and allow for the detection of flow fields in the bioresearch and chemical fields.

\section{Acknowledgement}

This research was supported by KAKENHI 19K04224.

\section{References}

1. T. Chován and A. Guttman, Trends Biotechnol., 20 (2002) 116.

2. T. M. Squires and S. R. Quake, Rev. Mod. Phys., 77 (2005) 977.

3. Z. G. Mills, W. Mao, and A. Alexeev, Trends Biotechnol., 31 (2013) 426.

4. B. Cetin, M. B. Özer, and M. E. Solmaz, Biochem. Eng. J., 92 (2014) 63.

5. D. E. Patabadige, S. Jia, J. Sibbitts, J. Sadeghi, K. Sellens, and C. T. Culbertson, Anal. Chem., 88 (2016) 320.

6. J. P. Brody, P. Yager, R. E. Goldstein, and R. H. Austin, Biophys. J., 71 (1996) 3430.

7. S. Weiße, M. Heydt, T. Maier, S. Schulz, J. P. Spatz, M. Grunze, T. Haraszti, and A. Rosenhahn, Phys. Chem. Chem. Phys., 13 (2011) 13395.

8. J. Garcia-Sucerquia, W. Xu, S. K. Jericho, M. H. Jericho, and H. J. Kreuzer, Optik, 119 (2008) 419.

9. M. Kuniyasu, Y. Aoyagi, N. Unno, S. Satake, K. Yuki, and Y. Seki, Opt. Rev., 23 (2016) 529.

10. N. Unno, S. Nakata, S. Satake, and J. Taniguchi, Exp. Fluids, 57 (2016) 120.

11. R. Budwig, Exp. Fluids, 17 (1994) 350.

12. A. Ravasio, S. Vaishnavi, B. Ladoux, and V. Viasnoff, Acta Biomater, 14 (2015) 5360.

13. J. Haisma, M. Verheijen, K. Van Den Heuvel, and J. Van Den Berg, J. Vac. Sci. Technol. B, 14 (1996) 4124.

14. H. Schift, J. Vac. Sci. Technol. B, 26 (2008) 458.

15. N. Unno, H. Kigami, T. Fujinami, S. Nakata, S. Satake, and J. Taniguchi, Microelectron. Eng., 180 (2017) 86. 
16. Y. Otsuka, S. Hiwasa, and J. Taniguchi, Microelectron. Eng., 123 (2014) 192.

17. N. Koo, J.W. Kim, M. Otto, C. Moormann, and H. Kurz, J. Vac. Sci. Technol. B, 29 (2011) $06 \mathrm{FC} 12$.

18. T. Uchida, F. Yu, M. Nihei, and J. Taniguchi,
Microelectron. Eng., 153, (2016) 43.

19. S. Satake, T. Kunugi, K. Sato, T. Ito, H. Kanamori, and J. Taniguchi, Meas. Sci. Technol., 17 (2006) 1647.

20. H. Li, R. Sadr, and M. Yoda, Exp. Fluids, 41 (2006) 185. 